\title{
Problem of Total Harmonic Distortion Measurement Performed by Smart Energy Meters
}

\author{
Piotr Kuwałek, Grzegorz Wiczyński \\ Institute of Electrical Engineering and Electronics, Faculty of Control, Robotics and Electrical Engineering, Poznan University \\ of Technology, Piotrowo Street 3A, 60-965, Poznan, Poland, piotr.kuwalek@put.poznan.pl
}

Currently, electricity is treated as commodity that should be delivered from a distributor to a consumer with a certain quality. The power quality is defined by the set of measures with specific limit values. One of the basic measures is the Total Harmonic Distortion (THD), which allows to assess the level of the voltage distortion. The measurement of THD ratio should be carried out in accordance with the normative specification. It is assumed that this requirement is met by class A power quality analyzers. Currently, measures are taken to monitor power quality in a large number of measurement points with the use of smart energy meters that are part of the Advanced Metering Infrastructure (AMI). The paper presents the problem of THD ratio measurement by AMI meters if voltage fluctuations occur. In such situation, inconsistency in measurement results of AMI meters and class A power quality analyzers occurs. The problem is presented on the basis of laboratory study results in which disturbances in power grid are recreated.

Keywords: harmonics, Total Harmonic Distortion (THD), power quality, voltage distortion, voltage fluctuation, AMI smart energy meters.

\section{INTRODUCTION}

The power quality in power grids is assessed by comparing measurement results of specific measures with limit values specified in country-specific standards and legal documents. From a consumer point of view, the power quality is assessed on the basis of incorrect operation or failure of loads used. One of the standards often referred to in the applicable legal documents is the standard IEC 50160 [1] (IEC - International Electrotechnical Commission). This standard defines specific measures that define the power quality, along with their acceptable limit values. Indicated specific measures include: fundamental frequency (power frequency), rms value of voltage, short-term $P_{s t}$ [2] and long-term $P_{l t}$ [3] flicker indicator, negative sequence symmetrical component, power factor, voltage individual harmonics, and Total Harmonic Distortion (THD) (THD ratio) [4]. Most often, a deterioration of power quality occurs as a result of voltage distortion or voltage fluctuation [5]. Currently, the voltage distortion level is defined by values of individual harmonics or by total measure, i.e. THD ratio [1], [6], [7], [8], [9]. In turn, a severity of voltage fluctuation (the level of flicker severity [10] caused by voltage fluctuations [11]) is measured using the indicators $P_{s t} / P_{l t}$. It is worth noting that increasing number of power electronic and energy-saving devices supplied from the power grid results in increasing voltage distortion. Hence, it is important that THD measurements are made as accurately as possible [12], [13], [14], [15]. In practice, this purpose is realized by performing measurements in accordance with the relevant standard, which specifies the THD measurement method and the THD measurement uncertainty [6]. In Europe, the most commonly used standard is the standard IEC 61000-4-30 [6], which defines the measurement method and the limit uncertainties of measurement of power quality measures defined in the standard IEC 50160 [1]. For the measurement of voltage distortion level, the standard IEC 61000-4-30 [6] refers to the standard IEC 61000-4-7 [16]. In the case of exceeding acceptable values or incorrect operation of loads, actions are taken to locate and identify causes of power quality deterioration [17], [18], [19], [20], [21], [22]. The identification and localization process is usually preceded by measurements verifying the power quality at a specific point in the power grid. It is worth noting that in some countries (e.g., Poland [23]) there are legal requirements additionally forcing the installation of AMI energy meters (AMI - Advanced Metering Infrastructure) with power quality assessment functionalities for each power consumer. In such countries, results of measurements performed by AMI energy meters (if available) are the basis for the complaint of poor power quality. Therefore, it is important that measurements with AMI meters comply with the normative specification. It is worth noting that the later verification of measurement results recorded during disturbances is usually impossible, as disturbing phenomena may not occur during later verification measurements (no retrospection possibility). The common use of AMI energy meters results in the pursuit of a low price. The low 
price of the measuring system is often inconsistent with the expected measuring circuit quality. It can result in inconsistency of selected functionalities of AMI meters with the standard IEC 61000-4-30 [6]. An example of such inconsistency can be THD measurements according to the standard IEEE 1459-2010 [24] (implemented in most AMI meters with the possibility of power quality evaluation), which defines the THD measurement method that does not require significant performance of processor (IEEE - Institute of Electrical and Electronics Engineers).

The paper presents the comparison of measurement results of six AMI meters with power quality evaluation functionalities with measurement results of class A power quality analyzer. Three-phase AMI meters of two manufacturers from different countries were used in the research. In the research on THD measurement were considered four types of supply voltage:

- "pure cosine",

- typical distorted voltage occurring in the low voltage (LV) network "clipped cosine" and "sharpened cosine" [25], [26], [27],

- voltage fluctuation modeled using amplitude modulation (AM) - typical model for a stiff power grid [28], [29], [30], [31],

- fluctuation of distorted voltage [32], [33], [34] (the most common situation in a LV networks).

The carried out own research and available literature [25], [26], [27] show that "clipped cosine" is the most common type of voltage distortion in LV networks. The inconsistency of THD measurement by AMI energy meters and by class A power quality analyzer are presented based on carried out experimental studies. The inconsistency of THD measurement increases with the increasing voltage fluctuation severity. The presented inconsistency in THD measurements shows the need to standardize voltage distortion assessment with the use of THD ratio in the process of power quality evaluation.

\section{PRINCIPLES OF THD MEASUREMENT}

Currently, only voltage and current are directly measured in most instruments used in the process of power quality evaluation (e.g., power quality analyzers, AMI energy meters). These signals are conditioned, low-pass filtered using an anti-aliasing filter, and then converted from analog to digital form. On the basis of obtained values of voltage/current samples, specific measures are determined (including THD ratio) by microcontroller with the implemented measurement method [35]. In practice, three methods of THD measurement (specified in the relevant normative and legal documents) are used to assess the voltage/current distortion level.

The standard IEC 50160 [1] and applicable legal documents in some countries define THD ratio as:

$$
\mathrm{THD}=\sqrt{\sum_{h=2}^{40}\left(u_{h}\right)^{2}},
$$

where: $h$ is harmonic order, $u_{h}$ is the relative percentage value of contribution of $h$-th harmonic in relation to the fundamental component.

The standard IEC 61000-4-30 [6] defines methods and uncertainties of measurement of individual power quality measures. In the case of THD measurement, the standard IEC 61000-4-30 [6] refers to the standard IEC 61000-47 [16]. This standard specifies the method of THD measurement based on harmonics determined using Discrete Fourier Transform (DFT). Considering the non-stationary nature of supply voltage related to load changes in the power grid, the standard IEC 61000-4-7 [16] specifies the method of THD measurement using the subgrouping method (aimed at limiting the "spectrum leakage" phenomenon effects [16], [36], [37]) for the measured signal in a rectangular measurement window with a duration equal to $N \cdot T_{c}$, where $T_{c}$ is the fundamental period of measured signal and $N$ is the total number of fundamental periods in the measurement window. In the case of a power grid with a fundamental frequency of $f_{c}=1 / T_{c}=50 \mathrm{~Hz}$, the standard IEC 61000-4-7 [16] specifies $N=10$. DFT analysis result for the indicated measurement window has a resolution of $\Delta f=1 /\left(N \cdot T_{c}\right)$ [35], [38]. The method of THD measurement for class A power quality analyzers using the subgrouping method can be described by the relationship:

$$
\mathrm{THD}=\sqrt{\sum_{h=2}^{40}\left(\frac{\sum_{i=-1}^{1}\left(U_{C, N \cdot h+i}\right)^{2}}{\sum_{i=-1}^{1}\left(U_{C, N+i}\right)^{2}}\right)} \cdot 100 \%,
$$

where: $U_{C, k=N \cdot h+i}$ is the rms value of $k$-th DFT component with frequency $f_{k}$, which is a total multiple of frequency resolution $\Delta f$ of DFT analysis $f_{k}=k \cdot \Delta f$.

In the case of AMI smart energy meters, most manufacturers declare that the method of THD measurement in these instruments is carried out in accordance with the standard IEEE 1459-2010 [24]. It is worth noting that some manufacturers do not define the method of THD measurement. Hence, it is often necessary to conduct a comprehensive test AMI meters to determine the method of THD measurement. The standard IEEE 1459-2010 [24] defines THD ratio as:

$$
\mathrm{THD}=\sqrt{\frac{U^{2}-U_{1}^{2}}{U_{1}^{2}}} \cdot 100 \%,
$$

where: $U$ is the rms value of measured signal, $U_{1}$ is the rms value of fundamental harmonic.

In the case of only voltage distortion by higher harmonics (without voltage fluctuation) in the power grid, the results obtained using (1), (2) and (3) should be equal to each other. Hence, if there is no loss of synchronization by the measuring instrument, the results of measurement by class A power quality analyzers and by AMI meters should be consistent (excluding inconsistency resulting from imperfections of measurement chains). 


\section{Selected TEST Signals}

\subsection{State without disturbances - "pure cosine"}

The adopted test signal at this stage can be described by the relationship:

$$
u(t)=\sqrt{2} U_{c} \cos \left(2 \pi f_{c} t\right),
$$

where: $U_{c}$ is the rms value of signal, $f_{c}$ is the fundamental frequency of signal. In this research stage, THD measurement results were obtained for the acceptable deviations of rms value of voltage in LV networks [1], [39] and for the acceptable deviations of fundamental frequency (power frequency) [1], [39], i.e., $U_{c} \in\{207,230,253\} \mathrm{V}$ and $f_{c} \in$ $\{47,49.5,49.9,50,50.1,50.5,52\} \mathrm{Hz}$. The adopted test signal at this stage is shown in Fig.1.

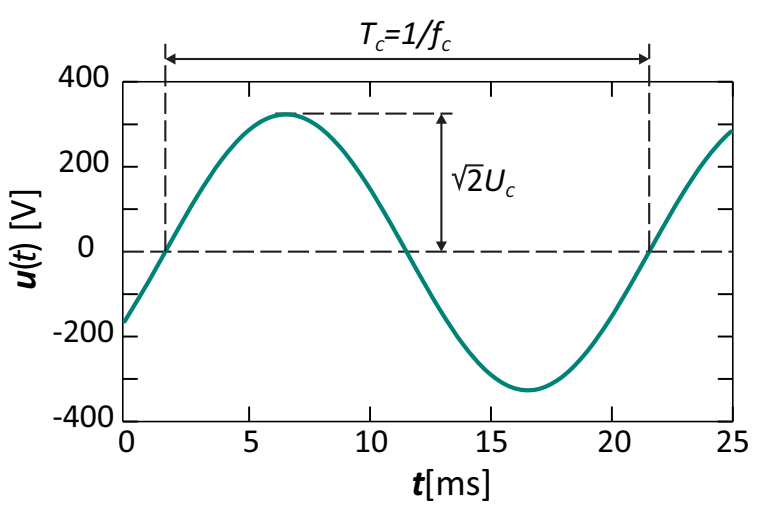

Fig.1. The exemplary waveform of "pure cosine".

\subsection{Voltage distorted by higher harmonics}

Two test signals were used in the research: "clipped cosine" and "sharpened cosine". The "clipped cosine" signal is a typical distortion of the phase voltage in LV networks, caused by the charging capacitors of switching power supplies. The "sharpened cosine" signal is a typical distortion of line-to-line voltage and is caused by the distortion of "clipped cosine" phase voltages.

The "clipped cosine" type signal is described by the relationship:

$$
u(t)=\left\{\begin{array}{ccc}
k_{U} m_{c} & \text { if } & \cos \left(2 \pi f_{c} t\right)>m_{c} \\
k_{U} \cos \left(2 \pi f_{c} t\right) & \text { if } & -m_{c} \geq \cos \left(2 \pi f_{c} t\right) \leq m_{c} \\
-k_{U} m_{c} & \text { if } & \cos \left(2 \pi f_{c} t\right)<-m_{c}
\end{array}\right.
$$

where: $f_{c}=50 \mathrm{~Hz} ; k_{U}$ is a scaling value which, for a given value of $m_{c}$, allows conversion of the rms value of test signal to the nominal value in $\mathrm{LV}$ networks, i.e. $230 \mathrm{~V} ; m_{c}$ defines the clipping level and is given by the equation:

$$
m_{c}=\frac{M_{c}}{M} \leq 1,
$$

where: $M_{c}$ and $M$ are amplitudes after and before clipping, respectively (see Fig.2.). An example of the adopted test signal of the "clipped cosine" type is shown in Fig.2.

The "sharpened cosine" signal can be described by the relationship:

$$
u(t)=u_{L 1}(t)-u_{L 2}(t)
$$

where: $u_{L 1}(t)$ is described by (5), and $u_{L 2}(t)$ is described by the relationship:

$$
u_{L 2}(t)=\left\{\begin{array}{ccc}
k_{U} m_{c} & \text { if } & \text { case } 1 \\
k_{U} \cos \left(2 \pi f_{c} t-\frac{2 \pi}{3}\right) & \text { if } & \text { case 2 } \\
-k_{U} m_{c} & \text { if } & \text { case 3 }
\end{array}\right.
$$

where:

$$
\begin{array}{lc}
\text { case 1: } & \cos \left(2 \pi f_{c} t-\frac{2 \pi}{3}\right)>m_{c} \\
\text { case 2: } & -m_{c} \geq \cos \left(2 \pi f_{c} t-\frac{2 \pi}{3}\right) \leq m_{c} . \\
\text { case 3: } & \cos \left(2 \pi f_{c} t-\frac{2 \pi}{3}\right)<-m_{c}
\end{array}
$$

An example of the adopted test signal of "sharpened cosine" type is shown in Fig.3.

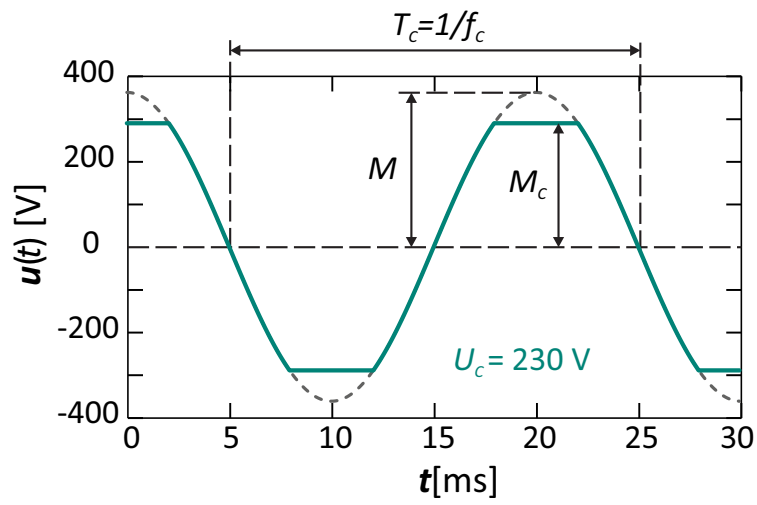

Fig. 2. The exemplary waveform of "clipped cosine" for $m_{c}=0.8$ and $k_{U} \approx 361.6$.

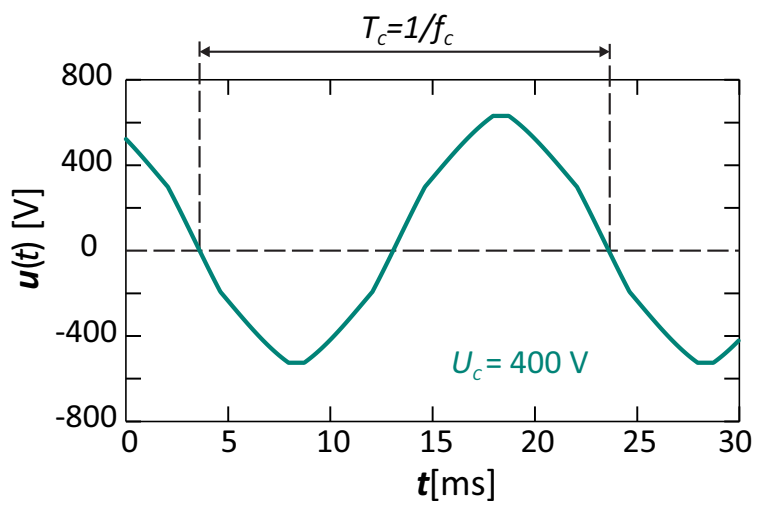

Fig. 3. The exemplary waveform of "sharpened cosine" with $m_{c}=$ 0.8 for $u_{L 1}(t)$ and $u_{L 2}(t)$.

\subsection{Voltage fluctuation}

The adopted test signal at this stage can be described by the relationship [40]:

$$
u(t)=u_{c}(t) \cdot[1+\overbrace{\left.\frac{1}{2\left(\frac{\Delta U}{U}\right) \frac{1}{100} \operatorname{sign}\left[\cos \left(2 \pi f_{m} t\right)\right.}\right]}^{\text {modulating signal }}],
$$


where: $u_{c}(t)$ is the carrier signal described by $(4),(\Delta U / U)$ is the modulation depth (relative amplitude of voltage fluctuation expressed in \%), $f_{m}$ is the frequency of modulating signal (frequency of voltage fluctuation). The adopted test signal is a typical voltage fluctuation in a stiff power grid, resulting from a cyclic switching on and off of loads. The dependence of THD measurement results on voltage fluctuation for the harmonic carrier signal is determined on the basis of obtained measurement results. The modulation depth $(\Delta U / U)$ and modulating frequency $f_{m}$ were chosen in such a way as to obtain the $P_{s t}$ value equal to 1 and 10, respectively. An example of the test signal described by (10) is shown in Fig.4.

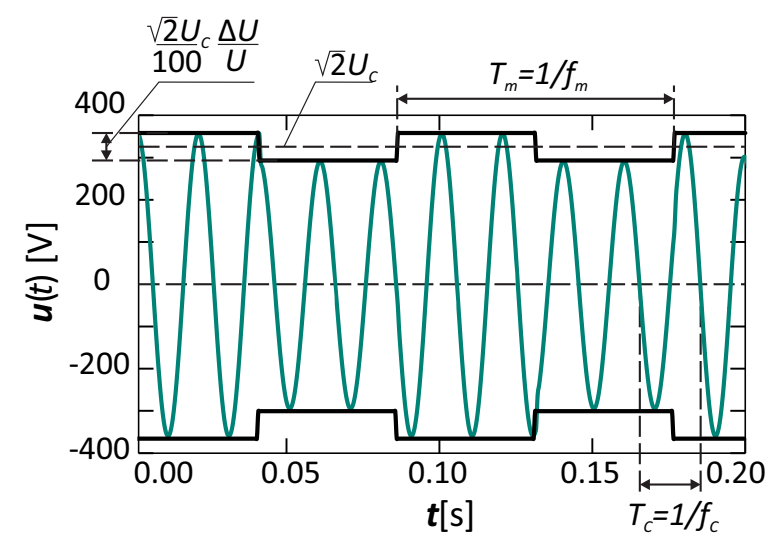

Fig. 4. The exemplary waveform of voltage fluctuation with "pure cosine" carrier wave.

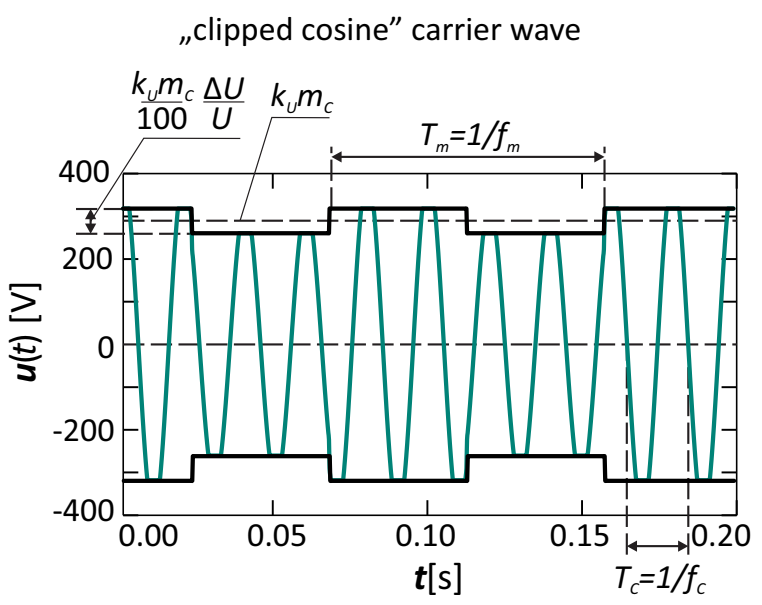

Fig.5. The exemplary waveform of voltage fluctuation with "clipped cosine" carrier wave.

\subsection{Fluctuation of distorted voltage}

The adopted test signal at this stage can be described by (10), where $u_{c}(t)$ is the signal described by (5) for $m_{c}=$ 0.8 . As previously mentioned, the test signal defined in this way represents a state often occuring in LV networks. The dependence of THD measurement results on voltage fluctua- tion for the distorted carrier signal is determined on the basis of obtained measurement results. The modulation depth $(\Delta U / U)$ and modulating frequency $f_{m}$ were chosen in such a way as to obtain the $P_{s t}$ value equal to 3 and 10, respectively. An example of the test signal described by (10) with distorted carrier wave is shown in Fig.5.

\section{RESEARCH DESCRIPTION}

The experimental laboratory studies were carried out in four stages. The following signals were considered at individual stages of research:

- "pure cosine" - state without disturbances;

- harmonic distortion (no voltage fluctuation);

- fluctuation of non-distorted ("pure cosine") voltage;

- fluctuation of distorted voltage (supply voltage distorted by higher harmonics).

The block diagram of laboratory setup is shown in Fig.6. In the research, test signals were generated in the following cascade: desktop computer - arbitrary generator (Agilent 33512B [41]) - power amplifier (built into a programmable AC power source Chroma 61502 [42]). The test signal was given to terminals of tested three-phase AMI meters and to terminals of class A power quality analyzer (PQA) (PQ Box 100 [43] - the THD measurement uncertainty determined on the basis of an uncertainty budget [44], [45] is $0.1 \%$ [36]). AMI meters were connected in such a way that the single-phase test voltage was given to three voltage terminals of each of the tested AMI meters. In this way, three measurement results were obtained from each of the tested AMI meters. The simultaneous measurement results were determined at 10-minute intervals. The measurement time for individual test signals was 20 minutes. Considering transient states related to the change of test signal [46], the first 10 minutes were rejected from the analysis. The analysis included the second 10-minute interval. Hence, during the research, nine results of THD measurements were obtained for each type of meter. The results of measurements by AMI meters were statistically evaluated by determining the maximum, minimum, and median values. The presentation of statistically processed measurement results of AMI meters is shown in Fig.7., where median values are connected by a line.

Additionally, for convergent results of measurements performed by AMI meters and by PQA, the characteristics of absolute and relative errors are determined in order to increase the clarity of presented experimental research results. The absolute error is determined according to the relationship:

$$
\Delta \mathrm{THD}=\left|\mathrm{THD}_{\mathrm{AMI}}-\mathrm{THD}_{\mathrm{PQA}}\right|,
$$

and the relative error is determined according to the relationship:

$$
\delta \mathrm{THD}=\frac{\left|\mathrm{THD}_{\mathrm{AMI}}-\mathrm{THD}_{\mathrm{PQA}}\right|}{\mathrm{THD}_{\mathrm{PQA}}} \cdot 100 \%,
$$


where: $\mathrm{THD}_{\mathrm{AMI}}$ is the THD ratio measured by the tested AMI meters, THD ${ }_{\mathrm{PQA}}$ is the reference value obtained from the PQA analyzer. It is worth noting that for the considered cases, the absolute and relative errors are expressed as a percentage.

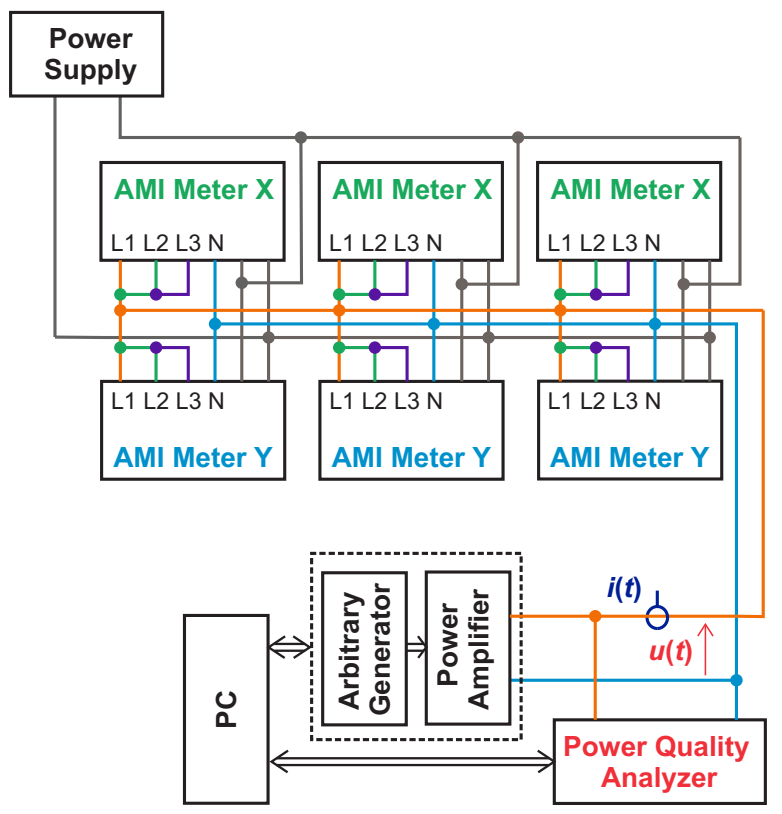

Fig.6. The block diagram of laboratory setup.

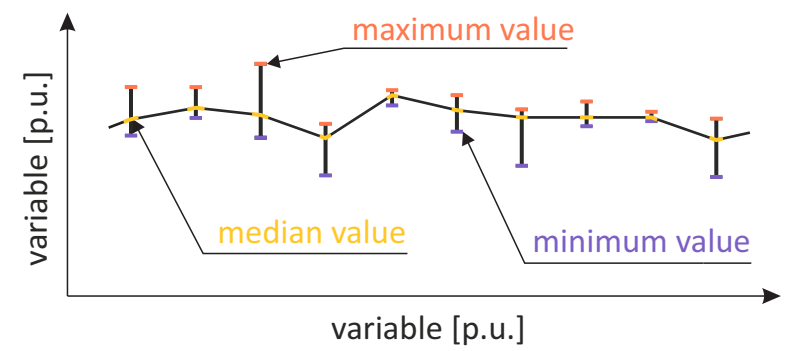

Fig.7. The method of presenting AMI meter measurement results.

\section{RESEARCH RESULTS AND DISCUSSION}

\subsection{State without disturbances - results and discussion}

The obtained results of tested AMI meters and PQA measurements for state without disturbances (see Section 3.1), are presented in Fig.8.

On the basis of obtained research results, it can be noticed that if the supply voltage is non distorted, then measurement results of AMI meters and the PQA analyzer are consistent. The minor inconsistency probably results from the different resolution of analog-to-digital converters in the measurement chains of individual instruments and the type of variable used in the microprocessor calculations. The theoretical THD ratio for the signal shown in Fig.1. is zero. The measured THD ratio is not zero due to imperfections in the test signal generation circuit. The THD ratio measured by PQA can be treated as a correct value and it can be assumed that the output voltage from the generation system has $\mathrm{THD} \approx 0.15 \%$.

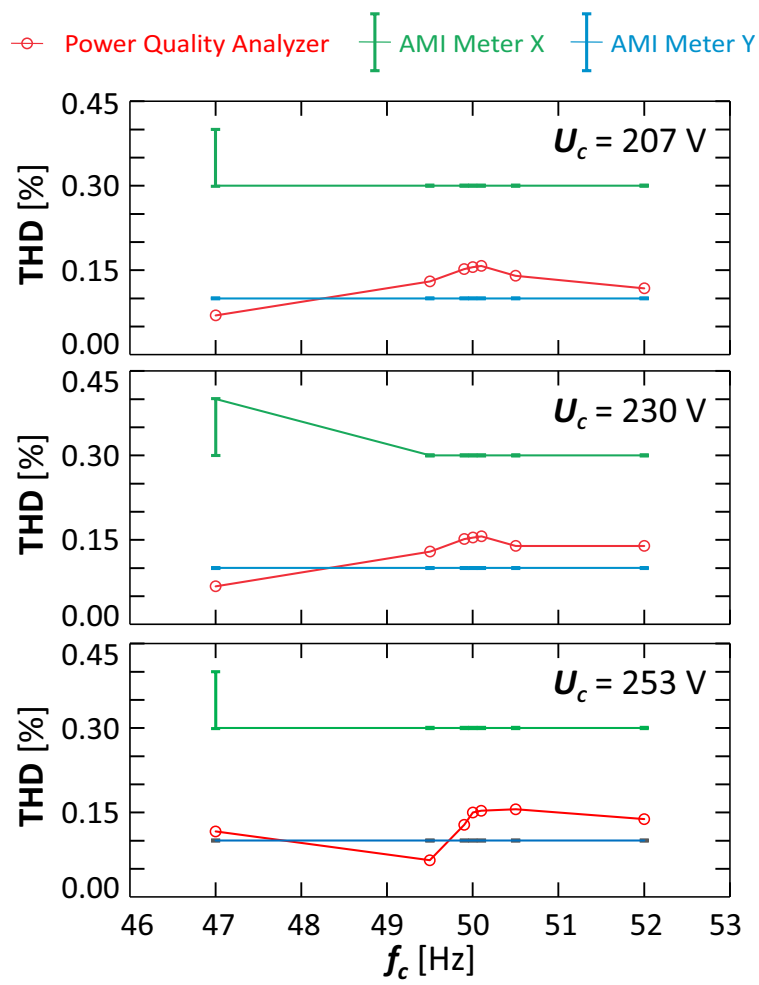

Fig. 8. The results of experimental studies for the "pure cosine" test signal.

\subsection{Voltage distorted by higher harmonics - results and dis- cussion}

THD measurement results for the "clipped cosine" type signal (see Section 3.2) are shown in Fig.9., and THD measurement results for the "sharpened cosine" type signal (see Section 3.2) are shown in Fig.10. The characteristics of absolute and relative errors of THD measurement by AMI meters as a function of $m_{c}$ are presented (determined using the relationship described by (11) and by (12)), to obtain the necessary clarity of presented research results.

On the basis of obtained research results, it can be notice that THD measurements by AMI meters and by the PQA analyzer are consistent in the case of voltage distorted by higher harmonics. The occurring inconsistency probably results mainly from the implementation of THD measurement method described by (3) that includes all harmonics up to the order determined by the bandwidth limitation in individual AMI meters, while the implementation of the THD measurement method described by (1) or (2) that includes only harmonics up to the order of 40 or 50 (depending on the normative requirements). The inconsistency is particularly noticeable for $m_{c} \rightarrow 0$, where the contribution of higher harmonics increases, the omission of which ((1) and (2)) causes a decrease in the measured THD ratio value. On the other hand, for $m_{c}=1$, there is no harmonic distortion. The absolute error $\triangle \mathrm{THD}$ is negligible. The increase in the relative 
error $\delta \mathrm{THD}$ is due to the fact that the reference value tends to 0 .

- Power Quality Analyzer I- AMI Meter X I AMI Meter Y

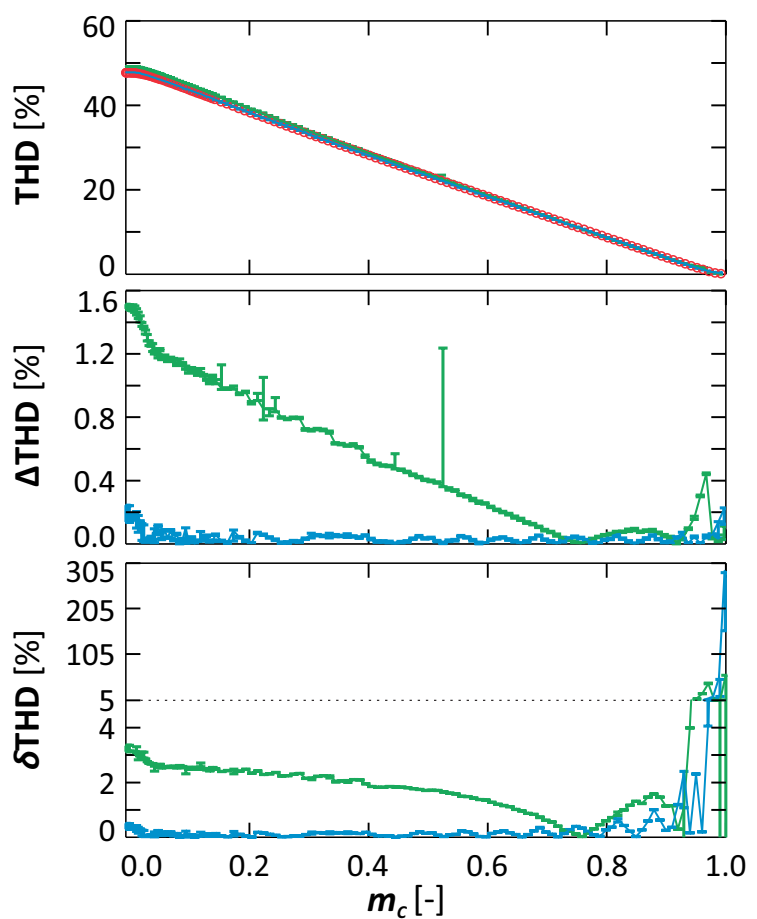

Fig. 9. The results of experimental studies for the "clipped cosine" test signal.
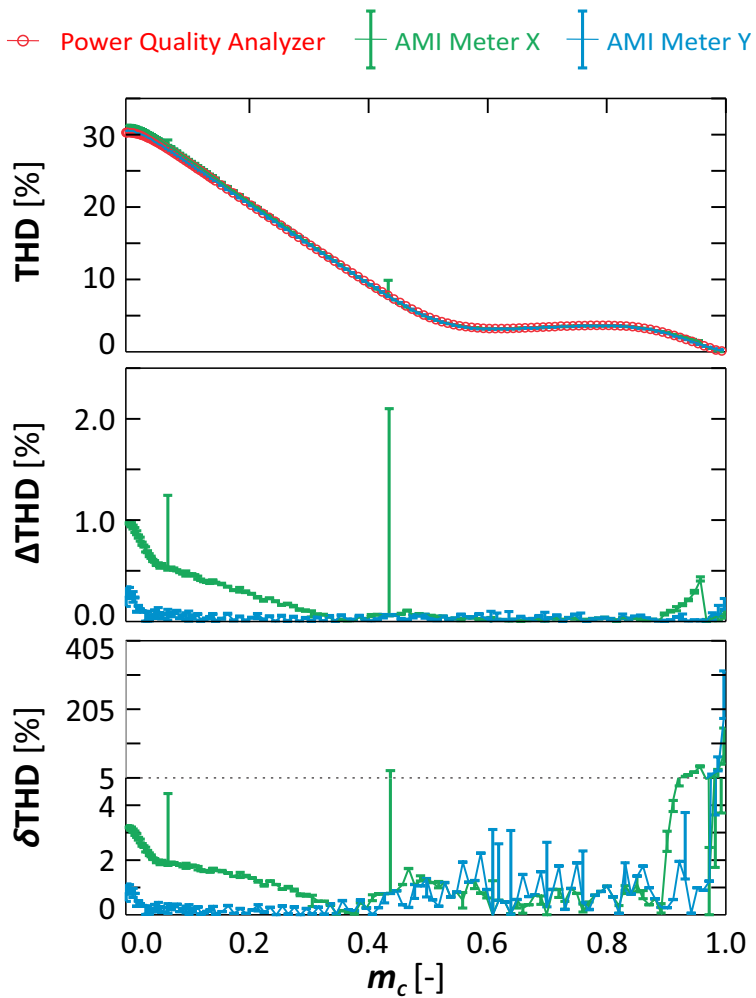

Fig. 10. The results of experimental studies for the "sharpened cosine" test signal.

\subsection{Voltage fluctuation - results and discussion}

The results of THD measurements by AMI meters and by the PQA analyzer in the case of only voltage fluctuation (see Section 3.3) are presented as the characteristics of THD $=\mathbf{f}\left(f_{m}, P_{s t}=\right.$ const. $)$ in Fig.11. and THD $=$ $\mathbf{f}\left((\Delta U / U), P_{s t}=\right.$ const. $)$ in Fig. 12 .

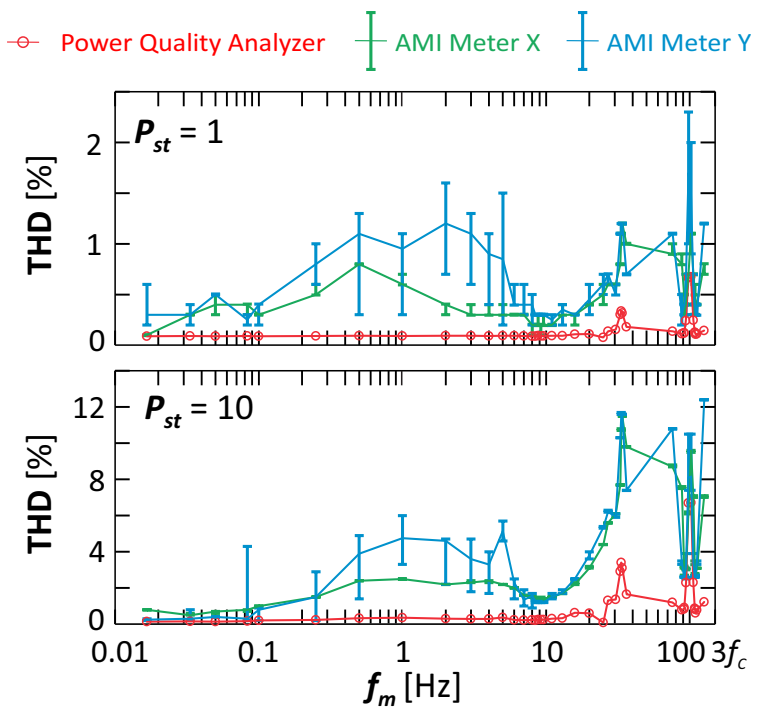

Fig. 11. The characteristics of THD $=\mathbf{f}\left(f_{m}, P_{s t}=1=\right.$ const. $)$ and $\mathrm{THD}=\mathbf{f}\left(f_{m}, P_{s t}=10=\right.$ const. $)$.
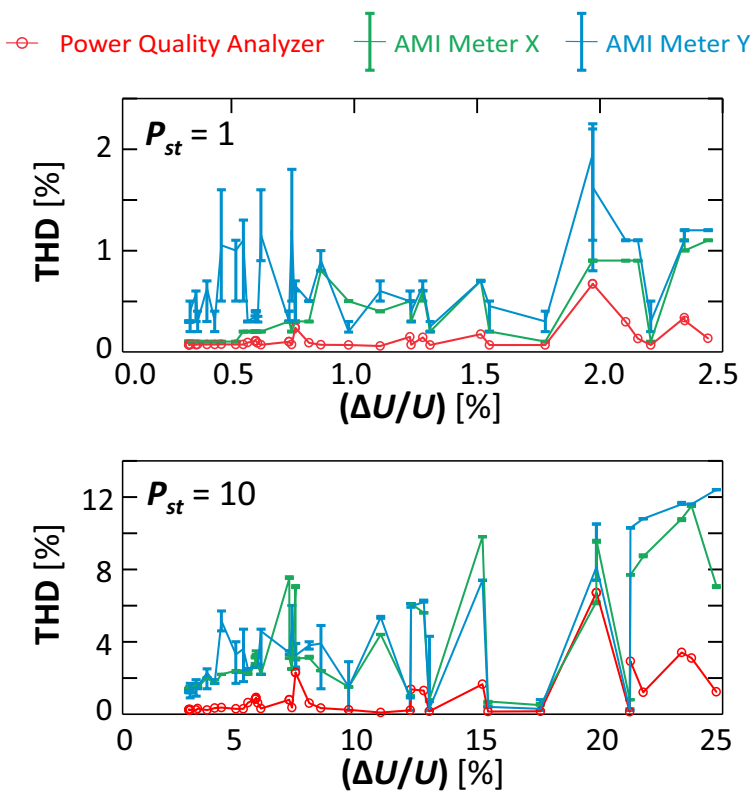

Fig. 12. The characteristics of $\mathrm{THD}=\mathbf{f}\left((\Delta U / U), P_{s t}=1=\right.$ const. $)$ and THD $=\mathbf{f}\left((\Delta U / U), P_{s t}=10=\right.$ const. $)$.

Analyzing Fig.11. and Fig.12., it can be noticed that the results of THD measurement by AMI meters and by the PQA 


\section{Std. IEC 50160, Legal Acts}

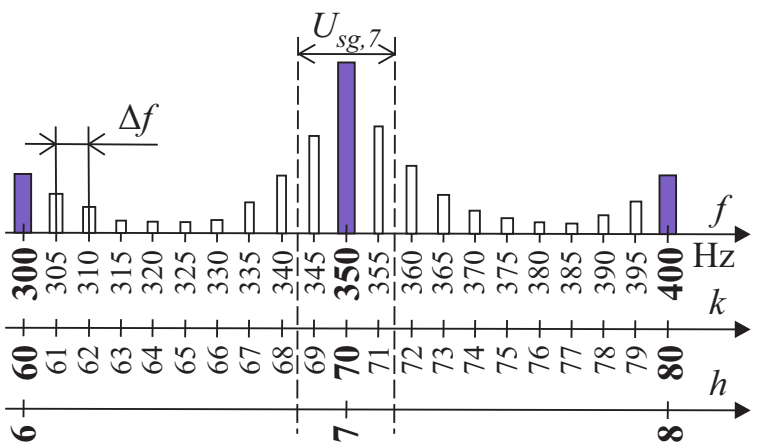

Std. IEC 61000-4-30, Class A Power Quality Analyzers

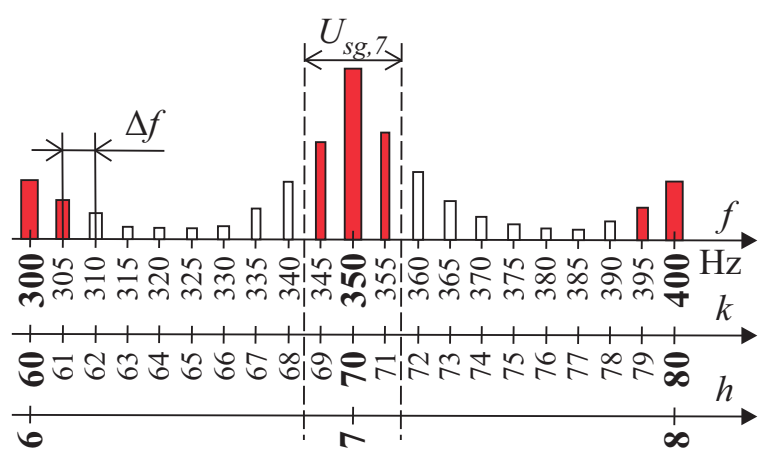

Std. IEEE 1459-2010, AMI Meter X, AMI Meter Y

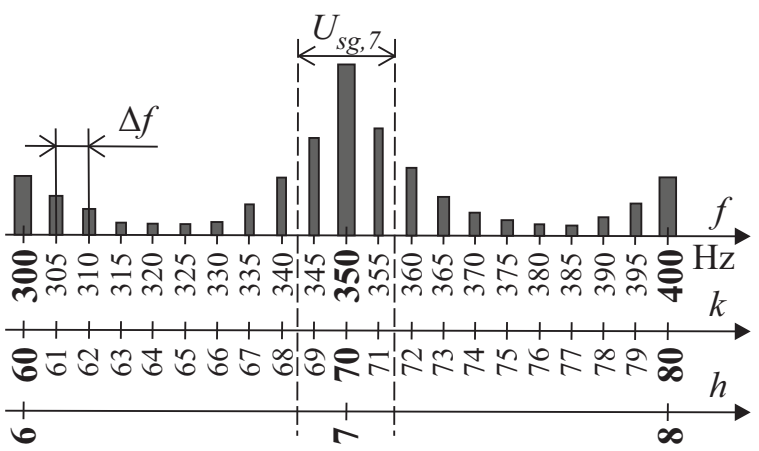

Fig. 13. The graphical explanation of THD measurement methods by individual instruments.

analyzer are not consistent in the case of voltage fluctuation occurrence. For the THD measurement errors described by (11) and (12), it can be noticed that the error of THD measurement by AMI meters increases with increasing voltage fluctuation severity and with increasing amplitude of voltage fluctuation. The theoretical THD ratio value given by (1) is zero for each case, such that $f_{m}$ is not the harmonic of carrier signal $u_{c}(t)$ (in this case the test signal represents the voltage distorted by higher harmonics).

THD ratio values measured by PQA are non-zero for modulating frequency close to total multiply of power frequency $f_{c}$, because the subgrouping algorithm described by (2) includes non-harmonic components. The inconsistency of results of THD measurement by AMI meters and by the PQA analyzer can be related to the differences in the measurement methods described by (2) and (3). Equation (3) includes, except harmonics and components close to harmonics, also subharmonics and interharmonics (in this research stage caused by voltage fluctuation). A graphic explanation of THD measurement methods by individual instruments is presented in Fig.13. For some cases there is the compliance of THD measurement by AMI meters and by the PQA analyzer. These cases occur when $f_{m} \rightarrow 0$ (no additional components in the determined signal spectrum for the basic measurement window used in the process of power quality evaluation). The inconsistency between the results of THD measurement by individual AMI meters probably results from the different bandwidth of individual AMI meters.

\subsection{Fluctuation of distorted voltage - results and discussion}

The results of THD measurements by AMI meters and by the PQA analyzer, in the case of simultaneous occurrence of voltage fluctuation and voltage distortion (see Section 3.4), are presented as the characteristics of THD $=\mathbf{f}\left(f_{m}, P_{s t}=\right.$ const. $)$ in Fig.14. and THD = $\mathbf{f}\left((\Delta U / U), P_{s t}=\right.$ const. $)$ in Fig. 15 .

Analyzing Fig.14. and Fig.15., it can be noticed that the results of THD measurement by AMI meters and by the PQA analyzer are not consistent in the case of simultaneous occurrence of voltage fluctuation and voltage distortion. For the THD measurement errors described by (11) and (12), it can
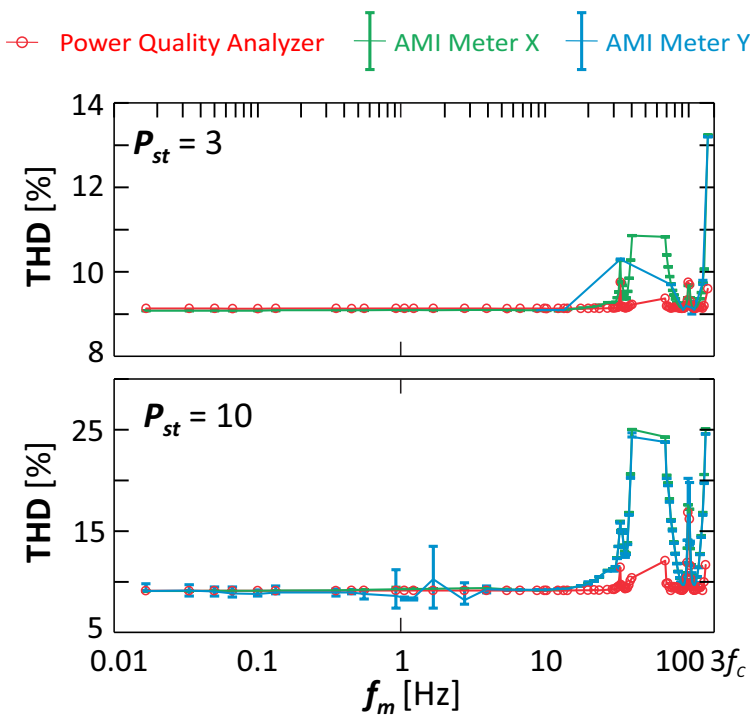

Fig. 14. The characteristics THD $=\mathbf{f}\left(f_{m}, P_{s t}=3=\right.$ const. $)$ and $\mathrm{THD}=\mathbf{f}\left(f_{m}, P_{s t}=10=\right.$ const. $)$ for "clipped cosine" carrier wave with $m_{c}=0.8$. 
be noticed that the error of THD measurement by AMI meters increases with increasing voltage fluctuation severity and with increasing amplitude of voltage fluctuation. The possible cause of inconsistency between results of THD measurements is the same as described in Section 5.3. For some cases there is the compliance of THD measurement by AMI meters and by the PQA analyzer. These cases occur when $f_{m} \rightarrow 0$ (no additional components in the determined signal spectrum for the basic measurement window used in the process of power quality evaluation).

$$
\text { - } \text { Power Quality Analyzer I AMI Meter X I AMI Meter Y }
$$
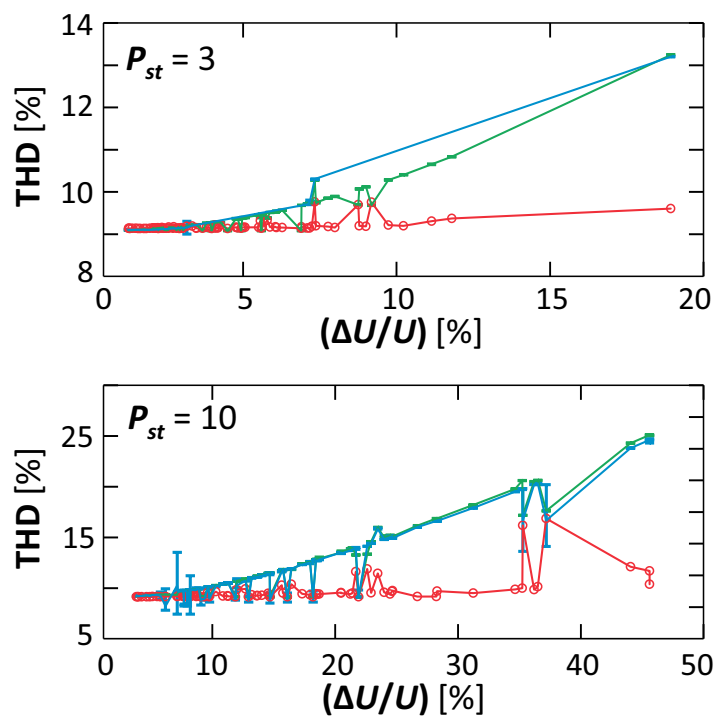

Fig. 15. The characteristics $\mathrm{THD}=\mathbf{f}\left((\Delta U / U), P_{s t}=3=\right.$ const. $)$ and THD $=\mathbf{f}\left((\Delta U / U), P_{s t}=10=\right.$ const. $)$ for "clipped cosine" carrier wave with $m_{c}=0.8$.
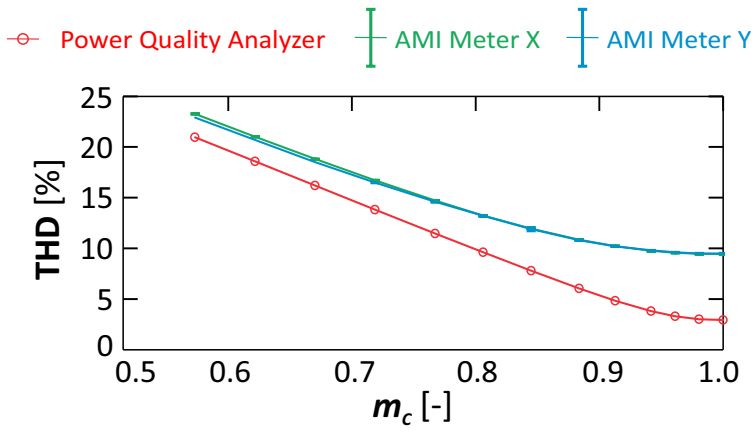

Fig. 16. The characteristic of THD $=\mathbf{f}\left(m_{c},(\Delta U / U)=18.9055 \%=\right.$ const., $f_{m}=135.55 \mathrm{~Hz}=$ const.) for "clipped cosine" carrier wave.

As part of this stage, the compliance of THD measurement was additionally examined depending on the distortion level (clipping level $m_{c}$ ) in the case of simultaneous occurrence of voltage fluctuation and voltage distortion. For this purpose, the test signal described by (10) was adopted, where the carrier signal $u_{c}(t)$ is the signal distorted by higher harmonics ("clipped cosine") given by (5) with $m_{c} \in[0.5 ; 1.0]$. The additional studies were performed for a constant modu- lation depth $(\Delta U / U)=18.9055 \%$ and for a constant modulating frequency $f_{m}=135.55 \mathrm{~Hz}$. For the adopted parameters, the obtained $P_{s t}$ value is equal to 3 . The results of additional studies from this stage for the "clipped cosine" carrier wave are presented in the characteristic THD = $\mathbf{f}\left(m_{c},(\Delta U / U)=\right.$ const., $f_{m}=$ const. $)$ in Fig.16. On the basis of the obtained results, we can notice the influence of distortion level (clipping level $m_{c}$ ) with simultaneous occurrence of voltage fluctuation with constant amplitude and frequency on THD measurement results.

\section{CONCLUSiOnS}

The paper presents the problem of THD measurement performed by commonly used AMI energy meters, considering real states occurring in the power grid (in particular in LV networks). When there is no disturbance or when there is voltage distortion caused by higher harmonics, THD measurements performed by AMI meters are consistent with THD measurements performed by instruments dedicated to power quality measurements (class A power quality analyzer). In the case of voltage distortion caused by higher harmonics, minor differences occur for voltage distortion with a significant contribution of higher harmonics, which is related to the limited harmonic order in implemented THD measurement method. In the case of voltage fluctuation (when the supply voltage is distorted or when the supply voltage is non distorted), there is the significant inconsistency in the results of THD measurement by AMI meters and by the class A power quality analyzer. The inconsistency of measurement results is probably due to used different THD definitions, which are not equal if voltage fluctuations occur. In this case, the THD ratio defined by the standard [24] includes not only the harmonics and spectrum components with a frequency close to the harmonic frequency (such as the THD ratio defined by the standard [16]), but also interharmonics and subharmonics. Considering new legal documents forcing the installation of AMI meters as the basic tool for monitoring the power quality, a significant problem occurs forcing the standardization of THD measurement methods. In particular, that the later verification of results of measurements performed by AMI energy meters using class A power quality analyzers can be impossible, because disturbing phenomena can-not occur during such control measurements (no possibility of retrospection).

\section{ACKNOWLEDGEMENT}

The work was supported by the Foundation for Polish Science (FNP) under Stipend START 45.2021.

\section{REFERENCES}

[1] IEC (2015). IEC Std. 50160: Voltage characteristics of electricity supplied by public distribution networks.

[2] IEC (2021)a. IEV number 161-08-18, short-term flicker indicator. http://www.electropedia.org/.

[3] IEC (2021)b. IEV number 161-08-19, long-term flicker indicator. http://www.electropedia.org/. 
[4] IEC (2021)c. IEV number 551-20-13, total harmonic ratio / total harmonic distortion. http://www.electropedia.org/.

[5] CEER (2016). 6th CEER Benchmarking Report on all the Quality of Electricity and Gas Supply 2016.

[6] IEC (2015). IEC Std. 61000-4-30: Testing and measurement techniques - Power quality measurement methods.

[7] Aiello, M., Cataliotti, A., Favuzza, S., Graditi, G. (2006). Theoretical and experimental comparison of total harmonic distortion factors for the evaluation of harmonic and interharmonic pollution of grid-connected photovoltaic systems. IEEE Transactions on Power Delivery 21(3), 1390-1397.

[8] Ruderman, A. (2015). About voltage total harmonic distortion for single- and three-phase multilevel inverters. IEEE Transactions on Industrial Electronics 62(3), 1548-1551.

[9] Collin, A., Djokic, S., Drapela, J., Langella, R., Testa, A. (2019). Proposal of a desynchronized processing technique for assessing high-frequency distortion in power systems. IEEE Transactions on Instrumentation and Measurement 68(10), 3883-3891.

[10] IEC (2021)a. IEV number 614-01-28, Flicker. http://www.electropedia.org/.

[11] IEC (2021)b. IEV number 161-08-05, voltage fluctuation. http://www.electropedia.org/.

[12] Antic, B., Mitrovic, Z., Vujicic, V. (2012). A method for harmonic measurement of real power grid signals with frequency drift using instruments with internally generated reference frequency. Measurement Science Review 12(6), 277-285.

[13] Bartman, J., Kwiatkowski, B. (2018). The influence of measurement methodology on the accuracy of electrical waveform distortion analysis. Measurement Science Review 18(2), 72-78.

[14] Espel, P., Poletaeff, A., Ndilimabaka, H. (2010). Traceability of voltage measurements for non-sinusoidal waveforms. Measurement Science Review 10(6), 200 204.

[15] ] Szolik, I., Kovac, K., Smiesko, V. (2003). Influence of digital signal processing on precision of power quality parameters measurement. Measurement Science Review 3(1), 35-38.

[16] IEC (2011). IEC Std. 61000-4-7: Testing and measurement techniques - general guide on harmonics and interharmonics measurements and instrumentation, for power supply systems and equipment connected thereto.

[17] Wiczynski, G. (2020). Determining location of voltage fluctuation source in radial power grid. Electric Power Systems Research 180, art. no. 106069.

[18] Kuwałek, P. (2021). Estimation of parameters associated with individual sources of voltage fluctuations. IEEE Transactions on Power Delivery 36(1), 351-361.

[19] Wei, S., Vanko, B., Kochan, O., Kochan, R., Su, J.
(2019). Improving monitoring of pulse distortions of voltage in power networks. Tekhnichna elektrodynamika 4, 65-69.

[20] Otomanski, P., Wiczynski, G. (2011). The usage of voltage and current fluctuation for localization of disturbing loads supplied from power grid. Przeglad Elektrotechniczny 87(1), 107-111.

[21] Lisowski, M., Masnicki, R., Mindykowski, J. (2019). Plc-enabled low voltage distribution network topology monitoring. IEEE Transactions on Smart Grid 10(6), 6436-6448.

[22] Chmielowiec, K., Wiczynski, G., Rodziewicz, T., Firlit, A., Dutka, M., Piatek, K. (2020). Location of power quality disturbances sources using aggregated data from energy meters. In: 2020 12th International Conference and Exhibition on Electrical Power Quality and Utilisation- (EPQU). pp. 1-5.

[23] Legal Act (2020). Act amending the Act - Energy Law and certain other Acts, Minister of Climate (formerly Minister of Energy).

[24] IEEE (2010). IEEE Std. 1459-2010 - IEEE Standard Definitions for the Measurement of Electric Power Quantities Under Sinusoidal, Nonsinusoidal, Balanced, or Unbalanced Conditions.

[25] Dyer, S., Dyer, J. (2011). Distortion: Total harmonic distortion in an asymmetrically clipped sinewave. IEEE Instrumentation Measurement Magazine 14(2), 48-51.

[26] Zhu, K., Sun, P., Zhou, L., Du, X., Luo, Q. (2020). Frequency-division virtual impedance shaping control method for grid-connected inverters in a weak and distorted grid. IEEE Transactions on Power Electronics 35(8), 8116-8129.

[27] Balasubramaniam, P., Prabha, S. (2015). Power quality issues, solutions and standards: A technology review. Journal of Applied Science and Engineering 18(4), 371380.

[28] Wu, C., Gao, Y., Li, C., Cao, Y., Iravani, R. (2020). Analytic mode decomposition and windowed three-point interpolated chirp-z transform for voltage flicker components detection. Electric Power Systems Research 186, art. no. 106382.

[29] KuwaL,ek, P. (2020). Am modulation signal estimation allowing further research on sources of voltage fluctuations. IEEE Transactions on Industrial Electronics 67(8), 6937-6945.

[30] Wiczynski, G. (2017). Estimation of pst indicator values on the basis of voltage fluctuation indices. IEEE Transactions on Instrumentation and Measurement 66(8), 2046-2055.

[31] Espel, P. (2010). Comparison of three accurate methods for flicker measurements. Metrologia 47(3), 287-294.

[32] Valtierra-Rodriguez, M., Romero-Troncoso, R., Osornio-Rios, R., Garcia-Perez, A. (2014). Detection and classification of single and combined power quality disturbances using neural networks. IEEE Transactions 
on Industrial Electronics 61, 2473-2482.

[33] Liu, H., Hussain, F., Shen, Y., Arif, S., Nazir, A., Abubakar, M. (2018). Complex power quality disturbances classification via curvelet transform and deep learning. Electric Power Systems Research 163, 1-9.

[34] Mahela, O., Shaik, A., Khan, B., Mahla, R., Alhelou, H. (2020). Recognition of complex power quality disturbances using s-transform based ruled decision tree. IEEE Access 8, 173530-173547.

[35] Otomanski, P., Wiczynski, G. (2015). The application of programmable ac source to generation of higher harmonics in examination of power quality. Przeglad Elektrotechniczny 91(8), 38-41.

[36] Kuwalek, P., Otomanski, P., Wandachowicz, K. (2020). Influence of the phenomenon of spectrum leakage on the evaluation process of metrological properties of power quality analyser. Energies 13(20), art. no. 5338.

[37] Kuwalek, P., Otomanski, P. (2019). The effect of the phenomenon of "spectrum leakage" on the measurement of power quality parameters. In: 2019 12th International Conference on Measurement. pp. 70-73.

[38] Testa, A., Gallo, D., Langella, R. (2004). On the processing of harmonics and interharmonics: using hanning window in standard framework. IEEE Transactions on Power Delivery 19(1), 28-34.

[39] Legal Act (2007). Decree of Ministry of Economy on detailed conditions of power system operation.
[40] Kuwalek, P. (2021). Influence of voltage variation on the measurement of total harmonic distortion (THD) by ami smart electricity meters. In: 2021 13th International Conference on Measurement. pp. 159-162.

[41] AWG (2012). Agilent 33500 series waveform generator: Operating and service uide.

[42] PA (2004). Programmable ac source 61501/61502/61503/61504 user manual.

[43] PQA (2011). Power quality analyser pq-box 100: Operating manual.

[44] Witkovsky, V., Wimmer, G., Durisova, Z., Duris, S., Palencar, R. (2017). Brief overview of methods for measurement uncertainty analysis: GUM uncertainty framework, monte carlo method, characteristic function approach. In: 2017 11th International Conference on Measurement. pp. 35-38.

[45] Dziarski, K., Hulewicz, A., Dombek, G. (2021). Lack of thermogram sharpness as component of thermographic temperature measurement uncertainty budget. Sensors 21(12), art. no. 4013.

[46] Majchrzak, J., Wiczyński, G. (2012). Basic characteristics of IEC flickermeter processing. Modelling and Simulation in Engineering 2012, art. no. 362849.

Received July 28, 2021. Accepted October 20, 2021. 